\title{
The Zeno effect and an inter-layer pairing mechanism for high-temperature superconductivity in layered materials
}

\author{
N. Kumar \\ Raman Research Institute, Bangalore 560080, India \\ E-mail: nkumar@rri.ernet.in
}

\begin{abstract}
Quantum Zeno Effect (QZE) is the suppression of the inter-subspace transition by a relatively fast intra-subspace decoherence. Earlier, we had proposed a QZE-based mechanism for the temperature-dependent normal-state c-axis resistivity of the layered high- $\mathrm{T}_{c}$ cuprate superconductors in which the singleparticle inter-layer tunneling is blocked by the strong intra-layer decoherence (entanglement). We now argue that while the single-particle inter-layer tunneling is thus blocked, the tunneling of the bosonic BCS-like pairs must remain unblocked inasmuch as a BCS pairing condensate is an eigenstate of the pair annihilation operator. This pair tunneling stabilizes high- $\mathrm{T}_{c}$ superconductivity energetically.
\end{abstract}

PACS No. 74.20.Mn; 74.20.Fg; 74.25.Fy

\section{INTRODUCTION}

At the very outset, let me state the general point of this work, which is that the normalstate c-axis resistivity is implicated in the high-temperature superconductivity of the layered cuprates. These high- $\mathrm{T}_{c}$ superconductors are by now well known to be qualitatively anisotropic in their normal-state kinetic properties. ${ }^{1-3}$ The qualitative anisotropy is most 
evident in their normal-state electrical resistivities - the out-of-plane resistivity $\rho_{c}(T)$ along the c-axis and the in-plane resistivity $\rho_{a b}(T)$ in the ab-plane - both in terms of their absolute magnitudes and the temperature dependences. Thus, $\rho_{c}(T)$ far exceeds the Mott-Ioffe-Regel (MIR) maximum metallic resistivity, with a transport mean-free path that works out to be much smaller than the inter-planar spacing. Still, it has a positive temperature coefficient of resistance $(T C R>0)$ at high temperatures, but develops a semiconductor-like resistive upturn as one approaches the $\mathrm{T}_{c}$. The $\rho_{a b}(T)$, on the other hand, has a sub-MIR metallic value with a positive TCR, and remains linear in $T$ down to about $\mathrm{T}_{c}$. So, the in-plane conduction is band-like, or relatively coherent (i.e., there are propagating, although much scattered, Bloch waves). In sharp contrast to this the c-axis transport is of the hoppingtype, or incoherent (the successive inter planar tunnelings are not phase correlated and one may not speak of the Bloch waves). The individual inter-layer tunneling is, however, phase coherent. Most importantly, at high temperatures, the c-axis resistivity tracks the ab-plane resistivity, i.e., $\Delta \rho_{c}(T) \propto \Delta \rho_{a b}(T)$ down to a temperature that decreases towards $T_{c}$ as the doping increases towards the optimal value corresponding to a maximum of $T_{c}$ within the given family. Indeed, for the optimally doped, high-quality untwinned single crystals, the Tlinear behaviour of $\rho_{c}(T)$ as well as that of $\rho_{a b}(T)$ has been reported to persist almost down to $T_{c}{ }^{4-5}$ This qualitative ly anisotropic electrical transport essentially involves the NonFermi Liquid (NFL) nature of the strongly correlated electronic system at hand, namely of the stacks of $2 \mathrm{D} \mathrm{CuO}_{2}$ sheets in the layered cuprates., ${ }^{3,6-8}$ The NFL behaviour is probed and revealed most clearly in the angle-resolved-photoemission spectra (ARPES) ${ }^{9}$ either by the striking absence of any sharp quasi particle peaks in the normal state (which, however, do reappear below the $\mathrm{T}_{c}$ ) or by the presence of a large incoherent tail to the quasi-particle peak as one moves away below the Fermi level. The latter is identifiable by the total disappearance of the quasi-particle peak. The near absence of the quasiparticle peak implies vanishing of the quasi-particle weight $Z_{k} \equiv\left(1-\partial R e \Sigma_{k}(w) / \partial w\right)_{w=\epsilon_{k}}^{-1}$ as $k \rightarrow k_{F}$, i.e., strong damping of the quasiparticle. This loss of coherence, and the consequent breakdown of the Landau FL picture, is also borne out by the optical conductivity in that the transport scattering 
rate is found to equal the quasi-particle energy. This forms the basis of our recent ${ }^{6,7}$ and the present work. We first demonstrate that this intra-planar NFL dynamics blocks the singleparticle tunneling between the weakly coupled ab-planes. ${ }^{6}$ Inasmuch as this suppression of the single-particle inter-planar tunneling may be viewed as blocking of the inter-subspace transitions due to intra-subspace coupling to the many-body environment (entanglement with the other-electronic degrees of freedom), we refer to our blocking mechanism as due to the Quantum Zeno Effect. ${ }^{10-12}$ Next we argue, admittedly heuristically, that this blocking mechanism may not be effective against the inter-planar tunneling of Cooper pairs for the planes prepared in the BCS-type trial pairing state, which, therefore, gets stabilized.

\section{BLOCKING OF THE SINGLE-PARTICLE INTERPLANAR TUNNELING: THE QUANTUM ZENO EFFECT}

First, consider a bilayer of two strongly-correlated NFLs, A and B, coupled weakly through the tunneling Hamiltonian (in obvious notation):

$$
H_{\perp}=-t_{\perp} \sum_{k \sigma}\left(b_{k \sigma}^{\dagger} a_{k \sigma}+h . c .\right), \ldots
$$

with $t_{\perp}>0$. The tunneling conserves the in-plane momentum.

Now, the change $\Delta E_{o}$ in the ground state energy of the bilayer due to this inter-planar tunneling can at once be written down by use of the Hellmann-Feynman charging theorem (For no change of symmetry):

$$
\Delta E_{o} \equiv E_{o}\left(t_{\perp}\right)-E_{o}(0)=\frac{2 t_{\perp}}{\pi} \sum_{k \sigma} \int_{0}^{\infty} d w \int_{0}^{1} d \eta \operatorname{Im} G_{\perp \eta}^{R}(k, w),
$$

where $G_{\perp \eta}^{R}(k, w)$ is the zero-temperature retarded Green function along the c-axis. Here $\Delta E_{o}(<0)$ gives the lowering of the ground state energy. 
Ignoring any vertex corrections to the inter-layer tunneling, we can express $G_{\perp \eta}^{R}(k, w)$ in terms of the intra-planar Green function $G_{\perp}^{R}(k, w)$, obtaining

$$
G_{\perp}^{R}(k, w)=\frac{\eta t_{\perp}\left(G_{\|}(k, w)\right)^{2}}{1-\eta^{2} t_{\perp}^{2}\left(G_{\|}(k, w)\right)^{2}}
$$

Thus, in this approximation the NFL nature enters through the in-plane Green function $G_{\|}^{R}\left(k_{1} w\right)$ only. We now use the semi-phenomenologically validated Marginal Fermi Liquid $(\mathrm{MFL})^{13}$ expression for $G_{\|}^{R}\left(k_{1} w\right)$ which is known to be consistent with a whole range of normal-state experimental facts on HTSC. This at once enables us to evaluate $\Delta E_{o}$ analytically. We find that the energy lowering, so calculated, due to the single particle tunneling (delocalization) along the c-axis sharply decreases as the MFL parameter characterizing the strength of the electron-electron interaction is increased. ${ }^{7}$ This clearly demonstrates that the intra-planar NFL character leads to an effective blocking of the single-particle tunneling along the c-axis, i.e., the single-particle tunneling matrix element $t_{\perp}$ is renormalized to a $t_{\perp e f f}^{(1)} \ll t_{\perp}$. This ties up neatly with the Caldeira-Leggett idea of dynamical c-axis localization due to intra-planar environmental couplin g. ${ }^{14}$ Note that the condition for the validity of our earlier proposal, namely that $\Delta \rho_{c}(T) \propto \Delta \rho_{a b}(T)$, now gets relaxed from $k_{B} T>t_{\perp}$ to

$k_{B} T>t_{\perp e f f}^{(1)}$. This, therefore, extends its domain of validity to much lower temperatures, obviating thus the concern expressed by some workers. ${ }^{15}$

\section{UNBLOCKED C-AXIS PAIR-TUNNELING AND SUPERCONDUCTIVITY}

For this, we again consider the weakly coupled bilayer comprising the planes A and B. Let the two many-body planar sub-systems be prepared in a trial state, namely, the BCS-like state $|\psi\rangle=|A>| B>$ with

$$
\left|A>=\Pi_{k}\left(u_{k}+v_{k} a_{k \uparrow}^{\dagger} a_{-k \downarrow}^{\dagger}\right) \equiv e^{g \alpha^{\dagger}}\right| 0>,
$$

and similarly for $\mid B>$. Here 


$$
g \alpha^{\dagger}=\sum_{k}\left(\frac{u_{k}}{i_{k}}\right) a_{k \uparrow}^{\dagger} a_{-k \downarrow}^{\dagger}
$$

and similarly for $g \beta^{\dagger}$. Thus $\alpha^{\dagger}(\alpha)$ and $\beta^{\dagger}(\alpha)$ are the pair creation (annihilation) operators for the two layers A and B respectively. Now, recall that for a dilute electronic system, and also, presumably, for a small pair-size (as is the case for the optimal hole doping levels in question), the pair operators are essentially bosonic and the coherent states $|A>| B>$, are eigenstates of the pair annihilation operators $\alpha, \beta$ respectively, with the eigenvalue $g$. Thus, a pair tunneling Hamiltonian

$$
H_{A B}^{(2)}=-t_{\perp}^{(2)}\left(\alpha^{\dagger} \beta+\beta^{\dagger} \alpha\right)
$$

would subtend a pair-tunneling amplitude along the c-axis

$$
<\psi\left|H_{A B}^{(2)}\right| \psi>=-2 t_{\perp}^{(2)}|g|^{2}
$$

This should imply that the pairs can tunnel adiabatically, a point missed in the other interlayer pairing theories. ${ }^{16,17}$

Crucial to our argument is the point that while the pair-tunneling is admittedly a twostep process $\left(t_{\perp}^{(2)}\right)$ involving a virtual intermediate single-particle tunneling $\left(t_{\perp}\right)$, we still have $T_{\perp}^{(2)} \sim t_{\perp}$. This is so because the intermediate state is nearly degenerate with the initial and the final states. Thus, we have the tunneling matrix elements: $t_{\perp}^{(2)} \sim t_{\perp} \gg t_{\perp e f f}^{(1)}$ This motivates us to considering a reduced Hamiltonian $h_{r e d}$ :

$$
h_{r e d}=h_{a}+h_{b}+h_{a b}^{(2)}+h_{a b}^{(1)}
$$

with

$$
\begin{aligned}
h_{a} & =\sum_{k, \sigma} \epsilon_{k} a_{k \sigma}^{\dagger} a_{k \sigma}+\frac{u}{2 N} \sum a_{k^{\prime} \uparrow}^{\dagger} a_{-k^{\prime} \downarrow}^{\dagger} a_{-k \downarrow} a_{k \uparrow} \\
h_{b} & =\sum_{k, \sigma} \epsilon_{k} b_{k \sigma}^{\dagger} b_{k \sigma}+\frac{u}{2 N} \sum_{k, k^{\prime}} b_{k^{\prime} \uparrow}^{\dagger} b_{-k^{\prime} \downarrow}^{\dagger} b_{-k \downarrow} b_{k \uparrow} \\
h_{a b}^{(2)} & =-\sum_{k, \sigma} t_{\perp}^{(2)}(k)\left(b_{k \sigma}^{\dagger} b_{-k-\sigma}^{\dagger} a_{k \sigma} a_{-k-\sigma}+h . c .\right) \\
h_{a b}^{(1)} & =-\sum_{k, \sigma} t_{\perp e f f}^{(1)}(k)\left(b_{k \sigma}^{\dagger} a_{k \sigma}+h . c .\right)
\end{aligned}
$$


with $u>0$ (repulsion). Here the usual Hubbard term with a strong on-site repulsion (ultimately responsible for the NFL nature) is replaced by a reduced pairing term (i.e., one which is repulsive, but still maintains the pairing condition) together with the pair tunneling term, and a residual, relatively suppressed single-particle tunneling term. Of course, for the cuprates all the inter-planar tunneling matrix elements have the angular (k) dependence $\propto\left(\cos a k_{x}-\cos a k_{y}\right)^{2}$, and hence dominant in the $(0, \pm \pi)$ and $( \pm \pi, 0)$ directions. This together with the short-ranged repulsion should favour a d-wave singlet pairing, as indeed is known to be the case.

The reduced Hamiltonian clearly supports a superconducting phase, at least at the mean field level. In order to see this in its essentiality, let us ignore the angular dependence of the tunneling matrix elements, and just look for an s-wave paired BCS-like state. Introducing the anomalous average $\Delta$ (assumed real):

$$
\Delta=\sum_{k}<a_{-k \downarrow} a_{k \uparrow}>=\sum_{k}<b_{-k \downarrow} b_{k \uparrow}>,
$$

the pair tunneling term reduces to

$$
h_{a b}^{(2)}=-\left(\frac{t_{\perp}^{(2)} \Delta}{N}\right) \sum_{\sigma, k}\left(b_{k \sigma}^{\dagger} a_{-k-\sigma}+\text { h.c. }\right),
$$

and similarly for the reduced Coulomb term containing $\mathrm{u}$.

The resulting bilinear Hamiltonian can now at once be diagonalized through a Bogoliubov transformation leading to a self-consistent gap equation for $\Delta$. Thus, for the simple case of $t_{\perp e f f}^{(1)}=0$, we get for the superconducting transition temperature $T_{c}$ (with $\gamma$ the Euler constant, $v$ the density of states per spin, and $W \sim$ hole Fermi-energy):

$$
k_{B} T_{c}=(4 \gamma / \pi) W \exp \left(-2 / \nu\left(t_{\perp}^{(2)}-u\right)\right)
$$

\section{CONCLUDING REMARKS}

I shall now conclude with a number of general remarks. The inter-layer pairing mechanism described above invokes the 2D-NFL (e.g., MFL) character for blocking the single- 
particle c-axis tunneling through entanglement with the intra-layer degrees of freedom the Quantum Zeno Effect. A quantum liquid with spin charge separation (e.g., a 2D Luttinger Liquid) seems to represent from our point of view an extreme case of such a blocking (confinement). $3,15,18$

The present mechanism, however, must be fully confronted with the pseudo-gap observed in the underdoped cuprates below a cross-over temperature $T^{*}\left(>T_{c}\right)$, with $T^{*}$ decreasing with increasing hole doping. ${ }^{19}$ The HTSC indeed seems to be a God of Gaps! More specif-

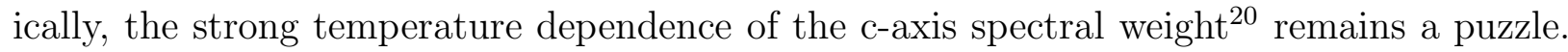
(The conductivity sum rule should be, of course, invariant under pairing, i.e., charge doubling mass doubling and electron-number halving, and temperature-insensitive for a proper choice of the frequency cut-off.)

Finally, we suggest that such qualitatively anisotropic materials exhibiting the Zeno Effective blocking of the c-axis single-particle tunneling be aptly called Zenophilic, and that the conditions for Zenophilicity, involving, e.g., the tunneling time, the life time and the Zeno time be examined carefully. ${ }^{21}$ 


\section{REFERENCES}

${ }^{1}$ Physical properties of high-temperature superconductors, Vols. I-III, ed. D.M. Ginsberg (World Scientific, Singapore, 1989-1992).

${ }^{2}$ B. Battlogg, in High-temperature superconductivity, The Los Alamos Symposium (1989), ed. K. Bedell et al. (Addison-Wesley, 1996),p. 37.

${ }^{3}$ P.W. Anderson, The theory of superconductivity in the high- $T_{c}$ cuprates, (Princeton University Press, Princeton, 1997).

${ }^{4}$ T.A. Friedmann et al., Phys. Rev. B42, 26 (1988).

${ }^{5}$ Y. Iye et al., Physica C 26, 153-155 (1988).

${ }^{6}$ N. Kumar and A.M. Jayannavar, Phys. Rev. B45, 5001 (1992); also, N. Kumar et al., Mod. Phys. Lett. B 11, 347 (1997); A.J. Leggett, Braz. J. Phys. 22, 129 (1992).

${ }^{7}$ N. Kumar et al., Phys. Rev. B57, 13399 (1998).

${ }^{8}$ Y. Zha, Phil. Mag. B 74, 497 (1996).

${ }^{9}$ T. Timusk and B. Statt, Rep. Prog. Phys. 62, 61 (1999).

${ }^{10}$ B. Misra and E.C.G. Sudarshan, J. Math. Phys. 18, 758 (1977).

${ }^{11}$ D. Home and M.A.B. Whittaker, Ann. Phys. (NY) 258, 237 (1997).

12 B. Nagels et al., Phys. Rev. Lett. 79, 3097 (1997).

${ }^{13}$ C.M. Varma et al., Phys. Rev. Lett. 63, 1996 (1989).

${ }^{14}$ A.J. Leggett et al., Rev. Mod.Phys. 50, 1 (1987).

${ }^{15}$ D.G. Clarke and S.P. Strong, Adv. Phys 46, 545 (1997).

${ }^{16}$ R. Zecchina, Mod. Phys. Lett. B 4, 1817 (1998).

17 Z. Z. Tisãnović, Phys. Rev. B36, R2364 (1987). 
18 J.M. Wheatley et al., Phys. Rev. B37, R5897 (1988).

${ }^{19}$ M. Randeria and J-C Campuzano, 1997 Preprint cond-mat/9709107.

${ }^{20}$ L.B. Ioffe and A.J. Millis, Science 285, 1241 (1999).

${ }^{21}$ L.S.Schulman, in Tunneling and its implications, eds. D. Mugnai, A. Ranfagni and L.S. Schulman (World Scientific, Singapore, 1997), p.121. 